University of Wollongong

Research Online

Faculty of Informatics - Papers (Archive)

Faculty of Engineering and Information

Sciences

$1-1-2009$

\title{
Modelling hydrogen adsorption within spherical, cylindrical and slit-shaped cavities
}

Aaron Thornton

University of Wollongong, awt23@uow.edu.au

James M. Hill

University of Wollongong, jhill@uow.edu.au

Follow this and additional works at: https://ro.uow.edu.au/infopapers

Part of the Physical Sciences and Mathematics Commons

\section{Recommended Citation}

Thornton, Aaron and Hill, James M.: Modelling hydrogen adsorption within spherical, cylindrical and slitshaped cavities 2009, 181-184.

https://ro.uow.edu.au/infopapers/1708

Research Online is the open access institutional repository for the University of Wollongong. For further information contact the UOW Library: research-pubs@uow.edu.au 


\title{
Modelling hydrogen adsorption within spherical, cylindrical and slit-shaped cavities
}

\author{
Abstract \\ The ability to store hydrogen in a highly dense state within absorbents via the mechanism of adsorption \\ has become a critical step in order to make a hydrogen powered automobile a practical reality. \\ Absorbents are composed of nano-scale cavities (or pores) designed in such a way that hydrogen will \\ adsorb onto the internal surface and thus be stored densely and safely enough for transport at ambient \\ conditions. This work investigates the effect of the geometry of the cavity shape by applying a new \\ mathematical model for predicting gas uptake, which is based on calculating the van der Waals' \\ interactions for hydrogen, within spherical, cylindrical and slit-shaped cavities and assuming equations of \\ state for hydrogen in bulk gas and adsorbed phase.

\section{Keywords} \\ Modelling, hydrogen, adsorption, within, spherical, cylindrical, slit, shaped, cavities

\section{Disciplines} \\ Physical Sciences and Mathematics

\section{Publication Details} \\ Thornton, A. W. \& Hill, J. M. (2009). Modelling hydrogen adsorption within spherical, cylindrical and slit- \\ shaped cavities. Advanced materials and nanotechnology Proceedings of the International American \\ Institute of Physics Conference (pp. 181-184). New York, USA: American Institute of Physics.
}




\title{
Modelling hydrogen adsorption within spherical, cylindrical and slit-shaped cavities
}

\author{
Aaron W. Thornton ${ }^{\mathrm{a}, \mathrm{b}, *}$ and James M. Hill ${ }^{\mathrm{a}}$ \\ ${ }^{a}$ Nanomechanics Group, School of Mathematics and Applied Statistics, \\ University of Wollongong, Wollongong, NSW, 2522, Australia \\ ${ }^{b}$ Commonwealth Scientific and Industrial Research Organization, \\ Material Science and Engineering, Locked bag 33, Clayton Sth MDC, Vic, 3169 Australia
}

\begin{abstract}
The ability to store hydrogen in a highly dense state within absorbents via the mechanism of adsorption has become a critical step in order to make a hydrogen powered automobile a practical reality. Absorbents are composed of nano-scale cavities (or pores) designed in such a way that hydrogen will adsorb onto the internal surface and thus be stored densely and safely enough for transport at ambient conditions. This work investigates the effect of the geometry of the cavity shape by applying a new mathematical model for predicting gas uptake, which is based on calculating the van der Waals' interactions for hydrogen, within spherical, cylindrical and slit-shaped cavities and assuming equations of state for hydrogen in bulk gas and adsorbed phase.
\end{abstract}

Keywords: hydrogen, storage, adsorption, gas, absorbent, cavity.

PACS: 68.43.-Mn, 61.46.-w, 61.48.-De

\section{INTRODUCTION}

As fossil fuel supplies diminish, the need for practical renewable energy grows [1]. The invention of the hydrogen electro-chemical fuel cell has brought the hope of a green future by creating electricity without the need for fossil fuels and producing pure steam as the exhaust product [2]. Two main obstacles for this technology to power automobiles are (I) the inability to collect pure hydrogen at low energy cost and (II) the inability to store hydrogen densely and safely enough for transport [1,3] and this paper is concerned with the latter issue.

The US Department of Energy (DOE) has set the targets for a gravimetric uptake of 6 wt. $\%\left(0.06 \mathrm{~kg} \mathrm{H}_{2}\right.$ / kg system) and a volumetric uptake of $0.045\left(\mathrm{~kg} \mathrm{H}_{2} /\right.$ $\mathrm{L}$ system) by 2010 , and even higher targets are proposed for 2015 [4]. There are many materials being investigated that could possibly satisfy the DOE targets and amongst them are those that utilize the mechanism of physi-sorption (a.k.a. adsorption) including, zeolites, nanotubes, fullerenes, polymers, fiberglass and metal-organic frameworks [5]. These materials have in common open structures composed

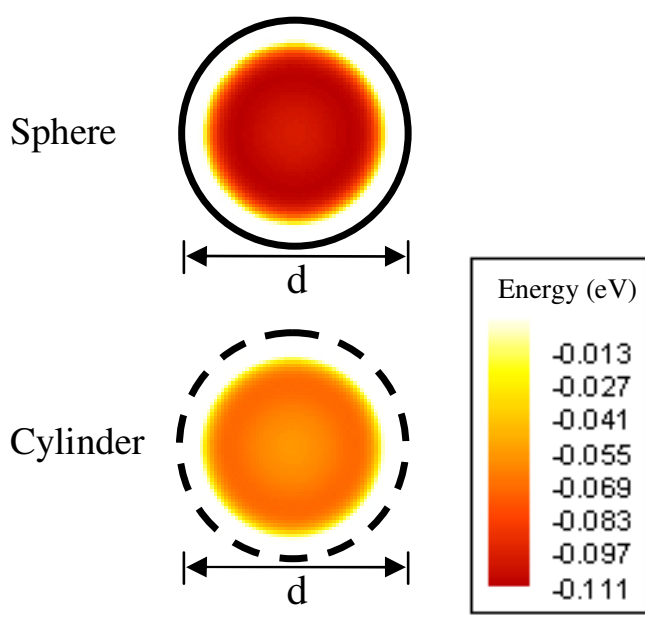

Slit

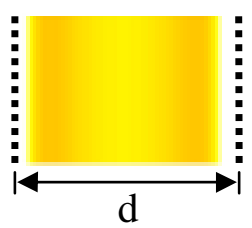

FIGURE 1. Potential energy for adsorption within cavities.

\footnotetext{
* Corresponding author. Tel.: +61-2-4221-3531; e-mail: aaron.thornton@ csiro.au.
} 
of nano-sized cavities (or pores, holes or channels) that provide adsorption sites for hydrogen to enter the material and adsorb onto internal surfaces. Hydrogen can be stored in a more dense fashion in the adsorbed phase as compared to bulk gas phase [6]. Thus, the objective is to design the porosity of the material so as to increase the amount of hydrogen in the adsorbed phase.

A key question which this paper addresses is, "what is the optimal cavity shape that achieves the highest amount of adsorption at close to ambient conditions?" Here we consider the most common geometric shapes, that is, spherical, cylindrical and slit-shaped. The method for prediction is a novel approach that incorporates the interactions between hydrogen and the cavity wall and is determined from the Lennard-Jones potential energy function, combined with two equations of state (one for the adsorbed phase and one for the bulk gas phase).

The following section provides the details for the model and the subsequent section presents the results. Finally, a conclusion is given outlining our major findings.

\section{METHOD}

The underlying physical concept of the model is that if the hydrogen molecule has a kinetic energy greater than the van der Waals binding energy with the cavity surface then the hydrogen molecule will be in the bulk gas phase. Conversely, if the hydrogen molecule has a kinetic energy less than the van der Waals binding energy with the cavity surface, then the hydrogen molecule will be in the adsorbed phase.

We begin by defining the van der Waals interactions between two atoms at a distance $\rho$ by the Lennard-Jones 6-12 potential energy function,

$$
\Phi(\rho)=-\frac{A}{\rho^{6}}+\frac{B}{\rho^{12}},
$$

where $\mathrm{A}$ and $\mathrm{B}$ are the attractive and repulsive constants, respectively, defined as $A=4 \varepsilon \sigma^{6}$ and $B=$ $4 \varepsilon \sigma^{12}$ ( $\sigma$ is the kinetic diameter and $\varepsilon$ is the well depth). Throughout this paper the Lennard-Jones constants for the interaction between hydrogen and carbon are used, derived from the Berthelot-Lorentz mixing rules, $\sigma=$ $3.159(\AA)$ ) and $\varepsilon / k=68.23(\mathrm{~K})$. Using the LennardJones function as a basis, continuum models for the potential energy between a particle and the internal surface of cylindrical, spherical and slit-shaped structures have been previously derived by integrating the interactions continuously across the whole surface (Cox and Hill [7]). The functions are given below and we refer the reader to $[7,8]$ for further details:
Cylindrical cavity:

$$
\begin{aligned}
& E_{c y l}(\rho)=\eta\left(-A H_{c y l}[3]+B H_{c y l}[6]\right), \\
& H_{c y l}[n]=\frac{2 \pi^{2} d^{2}}{(d)^{2 n}(2 n-2) !} \sum_{j=0}^{\infty}\left(\frac{\rho^{j}(2 n+2 j-2) !}{(2 d)^{j} j !(n+j-1) !}\right)^{2} .
\end{aligned}
$$

Spherical cavity:

$$
\begin{aligned}
& E_{s p h}(\rho)=\eta\left(-A H_{s p h}[3]+B H_{s p h}[6]\right), \\
& H_{s p h}[n]=\frac{\pi d}{\rho(2-n)}\left(\frac{1}{(\rho+d / 2)^{n-2}}-\frac{1}{(\rho-d / 2)^{n-2}}\right) .
\end{aligned}
$$

Slit-shaped cavity:

$$
\begin{aligned}
E_{s l i}(\rho)= & \pi \eta\left(-\frac{A}{2(\rho+d / 2)^{4}}+\frac{B}{5(\rho+d / 2)^{10}}\right) \\
& +\pi \eta\left(-\frac{A}{2(d / 2-\rho)^{4}}+\frac{B}{5(d / 2-\rho)^{10}}\right) .
\end{aligned}
$$

where $\eta$ is the atomic surface density on the cavity wall set to 0.3182 (No. C atoms / $\AA^{2}$ ). The resulting potential energy within each cavity is demonstrated in Figure 1, with identical dimensions $d$. The potential energy is enhanced when the potentials overlap each other as a result of the surfaces arranged close to each other andlor the surfaces having concave curvature.

The next step in the method is to determine the amount of space within the cavity for which hydrogen will either be in the adsorbed phase or in the bulk gas phase. As previously explained, we assume that a hydrogen molecule is in adsorbed phase if its kinetic energy is less than the van der Waals potential energy (binding energy) and vice-versa for the bulk gas phase. Therefore the free volume for adsorption is found by integrating the Arrhenius probability term (1-exp[$E / R T]$ ) over the total free volume within the cavity,

$$
V_{a d}=\int_{V_{f}} 1-\exp [-|E(\rho)| / R T] d \rho .
$$

Similarly, the free volume for the bulk gas,

$$
V_{\text {bulk }}=\int_{V_{f}} \exp [-|E(\rho)| / R T] d \rho .
$$

The total free volume $V_{f}$ is defined as the volume for which the potential energy is less than zero. In other words, the boundaries of this free volume are located where the potential energy is zero.

The final step in our method is to determine the number of hydrogen molecules in each phase. This is accomplished by using the equation of state. Firstly, 
the amount of molecules in adsorbed phase is found by solving a modified version of the Dieterici equation of state [9] for $n_{a d}$,

$$
P\left(V_{a d} / n_{a d}-v_{0}\right)=R T \exp (-|\bar{E}| / R T) .
$$

Similarly, the amount of molecules in the bulk gas phase is found by solving a simplified version of the van der Waals equation of state,

$$
P\left(V_{\text {bulk }} / n_{\text {bulk }}-v_{0}\right)=R T \text {. }
$$

where $P$ is pressure, $R$ is the universal gas constant, $T$ is temperature, $\bar{E}$ is the average potential energy for adsorption and $v_{0}$ is the occupied volume of closely packed hydrogen molecules. The total number of molecules are found from $n=n_{a d}+n_{b u l k}$, and the gravimetric uptake is calculated in the following way,

$$
w t . \%=\frac{n m}{(n m+M)} \times 100,
$$

where $m$ is the mass of a hydrogen molecule and $M$ is the mass of the cavity wall. In addition, the volumetric uptake is then calculated from,

$$
\bar{v}=\frac{n m}{V} \times 100,
$$

where $V$ is the total cavity volume (including space occupied by framework atoms).

\section{RESULTS AND DISCUSSION}

Since the US DOE objectives require operating conditions to be close as possible to the ambient temperature, the predicted hydrogen gravimetric and volumetric uptake at $200 \mathrm{~K}$ is shown in Figure 2 and Figure 3, respectively. We observe that the spherical cavity has the highest uptake at low pressures, followed by the cylindrical cavity at higher pressures and finally the slit-shaped cavity exceeds in uptake at extremely large pressures. This can be explained by the trade off which exists between the adsorption energy and the free volume [6]. Spherical shaped cavities have the highest potential energy for adsorption and therefore are capable of adsorbing large amounts of hydrogen at low pressures. However, as the pressure increases the hydrogen uptake is restricted by the amount of free volume available within the cavity. On the other hand, the cylindrical cavities have a lower adsorption energy but a higher free volume, and similarly, the slit-shaped cavities have an even lower adsorption energy but an even higher free volume. This behavior is enhanced at lower temperatures.

Figures 4 and 5 show the gravimetric and volumetric uptake, respectively, at a temperature of $243 \mathrm{~K}$ and a pressure of $100 \mathrm{~atm}$, which are the anticipated operating conditions for 2010 by the DOE. As expected at close to ambient conditions the slit-shaped cavity has the highest gravimetric uptake followed by the cylindrical cavity and the spherical cavity.

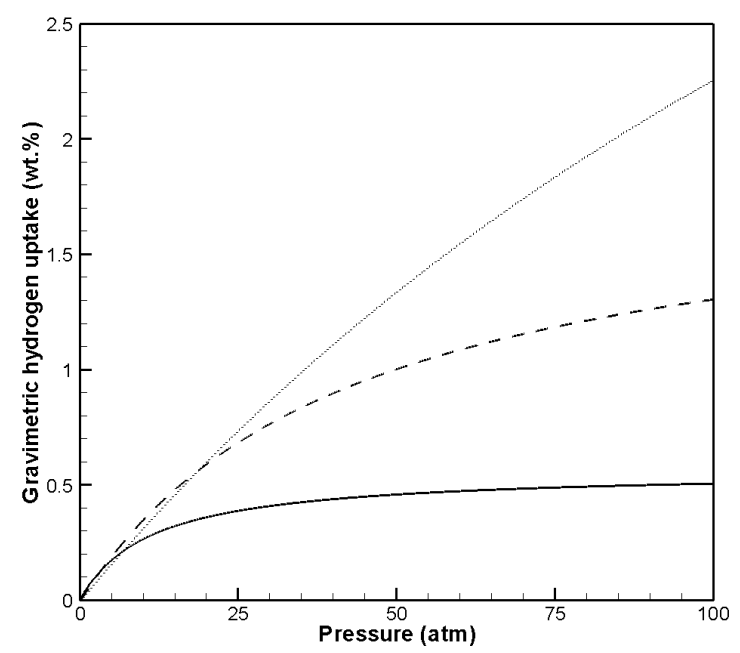

FIGURE 2. Hydrogen gravimetric uptake within spherical (solid), cylindrical (dashed) and slit-shaped (dotted) cavities of equal dimension $d=10 \AA$ at $200 \mathrm{~K}$.

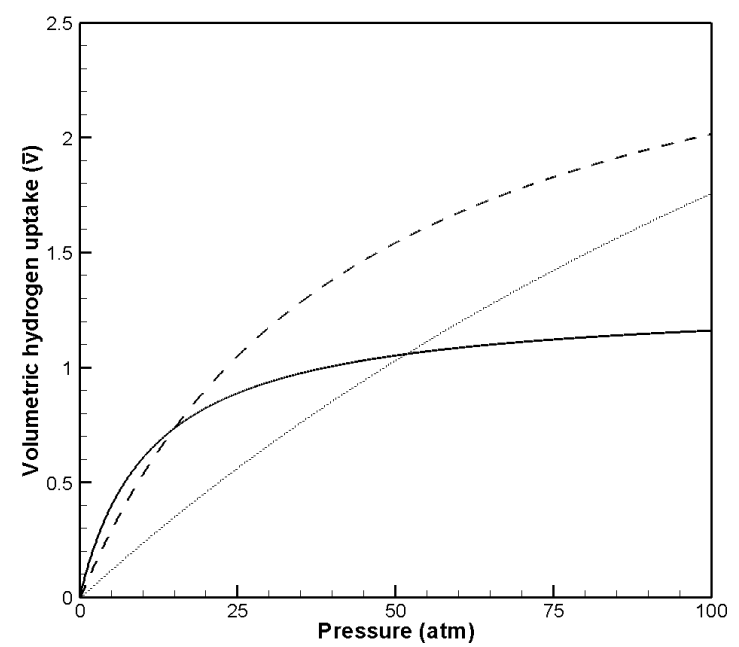

FIGURE 3. Hydrogen volumetric uptake within spherical (solid), cylindrical (dashed) and slit-shaped (dotted) cavities of equal dimension $d=10 \AA$ at $200 \mathrm{~K}$.

At these conditions, small amounts of hydrogen are in the adsorbed phase compared to the gas phase meaning that the ratio of cavity free volume $V_{f}$ over system mass $M$ is the dominant factor, observed in experimental and simulation work $[6,10]$. On the other hand, the cavity achieving the highest volumetric uptake depends upon the dimension of the cavity $d$. In this case, the ratio of the uptake amount $n$ over the total cavity volume $V$ is the dominant factor causing the amount of molecules in the adsorbed phase to play a crucial role in attaining maximum volumetric uptake at an optimal cavity dimension $d$. The optimal cavity size $d$ is $7.6 \AA$ for slit-shaped, $10.2 \AA$ for cylindrical 
and $13 \AA$ for spherical cavities. Slit-shaped cavities achieve higher volumetric uptake than cylindrical and spherical cavities at $100 \mathrm{~atm}$. However, at lower pressures the cylindrical cavities would achieve the highest followed by the spherical cavities at even lower pressures, as demonstrated in Figure 3. Therefore the effect of potential energy for adsorption, free volume and system mass on the total gravimetric and the volumetric uptake have been demonstrated.

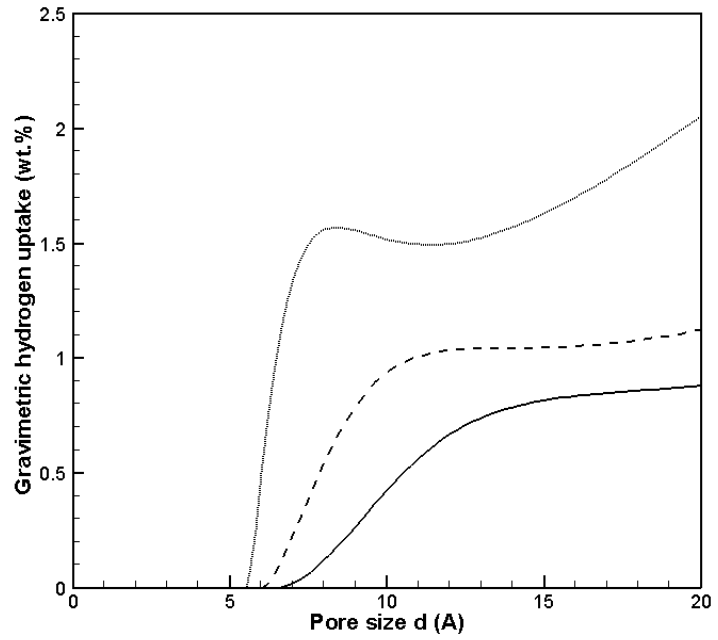

FIGURE 4. Hydrogen gravimetric uptake within spherical (solid), cylindrical (dashed) and slit-shaped (dotted) cavities with varying dimension $d$ at $243 \mathrm{~K}$ and $100 \mathrm{~atm}$.

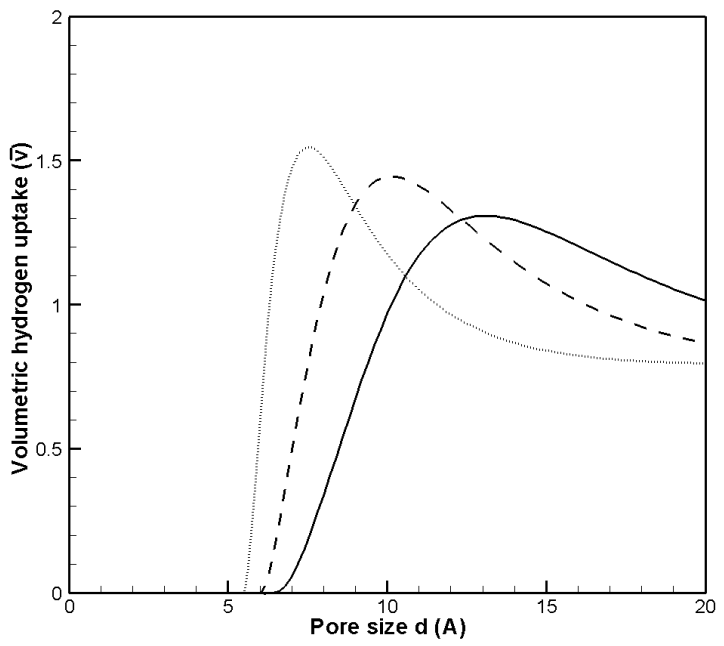

FIGURE 5. Hydrogen volumetric uptake within spherical (solid), cylindrical (dashed) and slit-shaped (dotted) cavities with varying dimension $d$ at $243 \mathrm{~K}$ and $100 \mathrm{~atm}$.

It is worth noting that these calculations do not take into account the interstitial gaps which exist when stacking cylinders or spheres together. With this as a consideration, the total uptake within the stack is expected to be lower than that predicted by this model. Spherical and cylindrical shaped cavities benefit from their concave surface where the energy potentials overlap each other creating a stronger potential, as also demonstrated by Okamoto and Miyamoto [11].

\section{CONCLUSION}

This work has investigated the hydrogen adsorption properties of spherical, cylindrical and slit-shaped cavities using a novel mathematical method based on fundamental principles. Since the operating conditions are desired to be as close to the ambient conditions for use within automobiles, the slit-shaped cavities show higher hydrogen uptake because of their large volume over mass ratio. At lower temperatures and lower pressure the spherical and cylindrical cavities absorb higher amounts of hydrogen due to the curved surfaces creating stronger adsorption energies.

\section{ACKNOWLEDGMENTS}

The authors wish to acknowledge support from the Australian Research Council and the Commonwealth Scientific and Industrial Research Organization. Special thanks are due to Matthew Hill for introducing the authors to this interesting topic.

\section{REFERENCES}

1. D. A. J. Rand and R. M. Dell, Hydrogen Energy: Challenges and Prospects. (Royal Society of Chemistry, Thomas Graham House, Science Park, Milton Road, Cambridge CB4 0WF, UK, 2008).

2. W. R. Grove, Philos. Mag. and J. Sci. 21, 417-420 (1842).

3. E. M. Gray, Adv. Appl. Ceram. 106, 25-28 (2007).

4. Multi-Year Research, Development and Demonstration Plan - Planned Program Activities for 2003-2010: Technical Plan; U.S. Department of Energy; http://www.eere.energy.gov/hydrogenandfuelcells/mypp/ pdfs/storage.pdf.

5. A. C. Dillon, K. M. Jones, T. A. Bekkedahl, C. H. Kiang, D. S. Bethune and M. J. Heben, Nature 386, 377-379 (1997).

6. H. Frost, T. Duren and R. Q. Snurr, J. Phys. Chem. B. 110, 9565-9570 (2006).

7. B. J. Cox, N. Thamwattana and J. M. Hill, J. Phys. AMath. Theor. 41, 27 (2008).

8. T. A. Hilder and J. M. Hill, J. Phys. A: Math. Theor. 40, 3851-3868 (2007).

9. C. Dieterici, Ann. Phys. Chem. Wiedemanns Ann. 69, 685 (1899).

10. M. Rzepka, P. Lamp and M. Casa-Lillo, J. Phys. Chem. B. 102, 10894-10898 (1998).

11. Y. Okamoto and Y. Miyamoto, J. Phys. Chem. B. 105, 3470 (2001). 
Copyright of AIP Conference Proceedings is the property of American Institute of Physics and its content may not be copied or emailed to multiple sites or posted to a listserv without the copyright holder's express written permission. However, users may print, download, or email articles for individual use. 